\title{
BIOGEOGRAFÍA Y SERIES DE VEGETACIÓN DE LA PROVINCIA DE MÁLAGA (ESPAÑA)
}

\author{
José Ma. NIETO CALDERA, Andrés PÉREZ LATORRE y Baltasar CABEZUDO
}

RESUMEN. Se ha realizado una sectorización biogeográfica de la provincia de Málaga, reconociéndose 7 sectores y 12 subsectores. Se han identificado 18 series de vegetación; 11 de carácter climatófilo (encinares, alcornocales, quejigares, pinsapares, melojares y sabinares) y 7 de carácter edafófilo (sabinares, pinares, pinsapares y acebuchales). Se describen dos subasociaciones nuevas: Chamaeropo humilis-Juniperetum phoeniceae genistetosum haenselerii y Daphno latifoliae-Aceretum granatensis quercetosum alpestris.

Palabras clave. Biogeografía, series de vegetación, vegetación climatófila, vegetación edafófila, Málaga, España.

SUMMARY. We have made a biogeographical division in the province of Málaga (Spain), recognizing seven sectors and twelve subsectors about which we have made an ecological and floristic diagnosis. It has been identified eighteen vegetation series: eleven climatophile ones (the woods and lands characterized by holm oak, cork oak, gall oak, Spanish fir, oak tree and juniper) and seven edaphophile ones (juniper lands, pine forests, relictic Spanish fir woods and wild olive tree groves). We also comment the sucessional communities and their environment relations. Two news subassociations are described through the text: Chamaeropo humilis-Juniperetum phoeniceae genistetosum haenselerii y Daphno latifoliae-Aceretum granatensis quercetosum alpestris.

Key words. Biogeography, vegetation series, climatophile vegetation, edaphophile vegetation, Málaga, Spain.

\section{INTRODUCCIÓN}

La provincia de Málaga, con una extensión de 7276 Km2 y un rango altitudinal que abarca desde el nivel del mar hasta los $2065 \mathrm{~m}$. está situada en el sur de la Península Ibérica (Andalucía). Por el Norte se abre al valle del Guadalquivir a través de la Depresión de Antequera; por el Oeste limita con las colinas del Campo de Gibraltar y la serranía de Cádiz y por el Este tiene frontera con la provincia de Granada

Trabajo realizado en base a la ayuda concedida por la Junta de Andalucía al grupo de investigación 4013. 
mediante la alineación montañosa de las sierras Tejeda y Almijara. Por el sur se pone en contacto con el mar Mediterráneo, al que se une a lo largo de los $180 \mathrm{Kms}$. de costa que posee.

Situada en el dominio de las Cordilleras Béticas, la provincia de Málaga se caracteriza por presentar un relieve muy compartimentado y una gran heterogeneidad fisiográfica. Geológicamente se pueden distinguir rocas sedimentarias $(79,6 \%$ de la superficie), metamórficas $(17,8 \%)$ e intrusivas $(2,6 \%)$. Los suelos son de tipo Litosols, Entisols, Vertisols, Inceptisols, Aridisols, Mollisols, Alfisols y suelos antropizados.

La red hidrográfica de la provincia está constituida fundamentalmente por ríos de corto recorrido en los que predomina el régimen estacional. Según datos de De la Rosa y Moreira (1987) presenta 2.951 cauces de primer orden y 1459 Ha. de carácter lagunar.

Se distinguen cuatro pisos bioclimáticos (Rivas-Martínez et al., 1991): termomediterráneo (0 / 500-800 m.), mesomediterráneo (500-800 / 1200-1400 m.), supramediterráneo (1200-1400 / 1850-1950 m.) y oromediterráneo (1850-1950 / 2065 m.). El ombroclima oscila de seco (Nerja, 424 mm.) a húmedo (Cartajima, 1288 $\mathrm{mm}$. .).

\section{BIOGEOGRAFÍA}

La sectorización biogeográfica de la provincia de Málaga ha sido tratada por diversos autores (Peinado Lorca y Rivas-Martínez, eds., 1987; Rivas-Martínez, 1987, 1988; Sainz Ollero y Hernández Bermejo, 1985; Salvo et al., 1983; Salvo y Cabezudo, 1984). Teniendo como base dicha información y los estudios corológicos, fitosociológicos y climáticos que hemos realizado adoptamos el siguiente esquema:

Región Mediterránea

Superprovincia Mediterráneo-Iberoatlántica

Provincia Bética

Sector Hispalense

Subsector Antequerano

Sector Rondeño

Subsector Rondense

Subsector Mijense

Subsector Torcalense

Sector Bermejense

Subsector Bermejense

Subsector Carratracense
Sector Malacitano-Axarquiense

Subsector Malacitano

Subsector Axarquiense

Sector Almijaro-Granatense

Subsector Almijarense

Sector Alpujarro-Gadorense

Subsector Alpujarreño

Sector Aljíbico

Subsector Aljíbico

Subsector Marbellí 


\section{Sector Hispalense}

El sector Hispalense ha sido tradicionalmente dividido en los subsectores Hispalense y Jerezano. El subsector Hispalense, en función de su heterogeneidad bioclimática, edáfica, litológica y florística lo consideramos separado en dos subsectores. El subsector Hispalense típico se correspondería con la Depresión del Guadalquivir, y el subsector ANTEQUERANO incluiría los territorios comprendidos entre dicho subsector y los territorios rondeños y subbéticos. El subsector Antequerano se caracteriza litológicamente por el predominio de margas y arcillas terciarias y triásicas, piso bioclimático mesomediterráneo cálido y un uso agrícola eminentemente olivarero. Comprende gran parte del norte de la provincia (Comarca de Antequera).

\section{Sector Rondeño}

Incluimos en este sector las sierras calcáreas y dolomíticas de la Serranía de Ronda y cordillera antequerana. Reconocemos tres subsectores: RONDENSE, el más representativo de este sector, caracterizado por un conjunto de sierras calizas, meso, supra y oromediterráneas; MIJENSE, agrupa el conjunto de sierras litorales termomediterraneas (meso), con ombroclima subhúmedo-húmedo y de naturaleza fundamentalmente dolomíticas con un grado alto de kakiritización y TORCALENSE, constituido por el conjunto de sierras mesomediterraneas (supra) que separan los sectores Hispalense y Malacitano-Axarquiense y en el que litológicamente predominan las calizas y el ombroclima es subhúmedo-húmedo

Son especies caracteristicas y/o diferenciales: Centaurea clementei Boiss. ex DC., Saxifraga reuterana Boiss., Anthyllis arundana Boiss. et Reuter, Arenaria arundana Gallego, Abies pinsapo Boiss., Armeria villosa subsp. villosa Girard, Erodium recoderi Auriault et Guittoneau, Erysimum rondae Polatschek, Galium pulvinatum Boiss., Helictotrichon filifolium subsp. arundanum Romero Zarco, Koeleria dasyphylla Willk., Linaria platycalix Boiss., Linaria oblongifolia (Boiss.) Boiss. et Reuter, Ononis saxicola Boiss. et Reuter, Saxifraga boissieri Engler, Sisymbrium arundanum Boiss., Armeria malacitana Nieto Feliner, Linaria clementei Boiss., Linaria huteri Lange, Teucrium chrysotrichum Lange, Genista haenseleri Boiss., Quercus alpestris Boiss., Nepeta amethystina subsp anticaria Cabezudo, Nieto Caldera et Navarro, Saxifraga biternata Boiss.

\section{Sector Bermejense}

Definido en base a la naturaleza litológica del sustrato, esencialmente constituidos por terrenos ígneos ultrabásicos (serpentinas, peridotitas). Es un sector con un área fragmentada geográficamente y fuertemente marcado por la presencia de una flora con numerosos edafoendemismos. Se reconocen dos subsectores, Bermejense, que ocuparía el núcleo mas extenso y meridional (sierras Bermeja, Real, Alpujata, etc.) con pisos bioclimáticos termo y mesomediterráneo (supra), suhúmedo-húmedo 
y CARRATRACENSE (sierra de Aguas), situado más al norte, con pisos termo y mesomediterráneo y ombroclima seco-subhúmedo.

Son especies características y/o diferenciales: Arenaria capillipes (Boiss.) Boiss., Arenaria retusa Boiss., Genista lanuginosa Spach, Galium viridiflorum Boiss. et Reuter, Halimiun atriplicifolium subsp. serpentinicola Rivas-Goday et RivasMartínez, Iberis fontqueri Pau, Staehelina baetica DC., Teucrium haenseleri Boiss., Allium rouy Gaut., Saxifraga gemmulosa Boiss., Silene fernandezii Jeanmonod.

\section{Sector Malacitano-Axarquiense}

En función de la diversidad litológica hemos dividido el tradicional sector Malacitano-Almijarense en dos sectores; el sector ALMIJARO-GRANATENSE caracterizado por la existencia de terrenos calizo-dolomíticos, y el MALACITANOAXARQUIENSE fundamentalmente constituido por terrenos paleozoicos y cuaternarios. El sector Malacitano-Axarquiense comprende las comarcas geográficas de la Axarquía, Montes de Málaga, Hoya de Málaga y Valle del Guadalhorce. Bioclimáticamente es predominante el piso termomediterráneo, que puede alcanzar cotas de hasta $1000 \mathrm{~m}$. y un ombroclima que oscila de seco a subhúmedo. Reconocemos dos subsectores, AXARQUIENSE (Montes de Málaga y Axarquía) predominando litologicamente esquistos y filitas y el subsector MALACITANO (Hoya de Málaga y Valle del Guadalhorce) con sustratos margosos y arcillosos.

\section{Sector Almijaro-Granatense}

Escasamente representado en la zona oriental de la provincia por el subsector ALMIJARENSE. Está formado por un conjunto de sierras dolomíticas variablemente kakiritizadas (sierras Tejeda y Almijara), con pisos bioclimáticos termo a oromediterráneo y ombroclima de seco a húmedo.

Son características y/o diferenciales la presencia de comunidades edafoxerófilas y numerosos edafoendemismos dolomitícolas como Anthyllis plumosa Domínguez, Anthyllis tejedensis Boiss., Arenaria delaguardiae López et Nieto Feliner, Arenaria racemosa Willk., Andryala agardhii Haenseler, Brachypodium boissieri (Boiss.) Nyman, Centaurea bombycina Boiss., Centaurea boissieri DC., Eryngium grosii Font Quer, Helianthemum viscidulum Boiss., Hieracium texedense Pau, Iberis grosii Pau, Linaria amoi Campo ex Amo, Nepeta granatensis Boiss., Pseudoscabiosa grossii (Font Quer) Devesa, Sideritis glacialis Boiss., Thymus longiflorus Boiss., Reseda paui subsp. almijarensis Valdés Bermejo et Kaercher, Teucrium fragile Boiss., Ulex parviflorus subsp. rivasgodayanus Cubas, Teucrium rotundifolium Schreber.

\section{Sector Alpujarro-Gadorense}

Puntualmente representado en la zona oriental de la provincia de Málaga por el subsector ALPUJARREÑO. Ocupa una estrecha banda litoral que partiendo desde 
Maro finaliza a la altura de la Cala del Moral. Litológicamente los sustratos son muy diversos, arcillas y margas neógenas y cuaternarias, filitas paleozoicas, calizas jurásicas, etc. Bioclimáticamente el piso es termomediterráneo inferior y el ombroclima seco.

Son especies características y/o diferenciales: Lavandula dentata L., Lavatera oblongifolia Boiss., Maytenus europaeus Boiss., Satureja cuneifolia subsp. malacitana (López) Cabezudo, Nieto Caldera et Navarro, Sideritis granatensis (Pau) Rivas Goday et González García, Sideritis pusilla var. salina Font Quer, Withania frutescens (L.) Pauquy.

\section{Sector Aljíbico}

Consideramos este sector incluido en la provincia BÉTICA a la espera de estudios más precisos en el noroeste de Marruecos (provincia Tingitana), donde podría situarse como sector ALJIBICO. Es un sector que ocupa el suroeste de la provincia de Málaga, definido fundamentalmente por la naturaleza acidófila de la vegetación que se asienta sobre cuarcitas (areniscas del aljibe) y esquistos; aparecen también importantes afloramientos de margas. Hemos distinguido el subsector ALJIBICO, con litología a base de areniscas cuarcíticas, piso bioclimático termo y mesomediterráneo y ombroclima subhúmedo-húmedo (hiperhúmedo) y el subsector MARBELLI, con esquistos, filitas y gneises paleozoicos, termomediterráneo y ombroclima subhúmedo.

Son especies características y/o diferenciales: Biscutella microcarpa DC., Cytisus tribracteolatus Webb, Satureja salzmannii P.W. Ball, Lamium flexuosum Ten., Teucrium scorodonia subsp. baeticum (Boiss. et Reuter) Tutin, Rhododendrom baeticum Boiss. et Reuter, Genista monspesulana (L.) L. Johnson, Cytisus villosus Pourret, Genista linifolia L.

\section{SERIES DE VEGETACIÓN}

A efectos tipológicos, paisajísticos y fisiográficos distinguimos dos tipos de series: climatófilas y edafófilas (Rivas-Martínez, 1985).

\section{Series climatófilas.}

Son numerosas las referencias existentes sobre la vegetación climatófila de la provincia de Málaga: Asensi y Díez Garretas (1988), Asensi y Rivas-Martínez (1976), Ceballos y Vicioso (1933), Díez Garretas, Cuenca y Asensi (1988), Martínez Parras et al. (1987), Nieto Caldera (1987), Nieto Caldera y Cabezudo (1988), Nieto Caldera, Pérez Latorre y Cabezudo (1990), Peinado Lorca y Rivas-Martínez, eds. (1987), etc. Las series presentes en la provincia son las siguientes: 
A. Encinares.

1. Smilaco mauritanicae-Querceto rotundifoliae $S$.

2. Paeonio broteroi-Querceto rotundifoliae $S$.

3. Berberido hispanicae-Querceto rotundifoliae $S$.

4. Adenocarpo decorticantis-Querceto rotundifoliae S.

B. Alcornocåles.

5. Myrto communis-Querceto suberis S.

6. Teucrio baetici-Querceto suberis $\mathrm{S}$.

C. Quejigares.

7. Rusco hypophylli-Querceto canariensis $S$.

8. Daphno latifoliae-Acereto granatensis $\mathrm{S}$.

D. Pinsapar.

9. Paeonio broteroi-Abieteto pinsapi S.

E. Melojar.

10. Adenocarpo decorticantis-Querceto pyrenaicae $S$.

F. Sabinar.

11. Daphno oleoidi-Pineto sylvestris $S$.

1. Serie termomediterránea, Bética, Algarviense y norteafricana, seca-subhúmeda de la encina (Quercus rotundifolia). Smilaco mauritanicae-Querceto rotundifoliae S.

Los encinares climatófilos termomediterráneos (Smilaco mauritanicaeQuercetum rotundifoliae) están representados en la provincia en forma de 4 faciaciones, consecuencia inmediata de la condición heterogénea de los biotopos donde se establece.

1.1. Faciación típica. Este encinar en la actualidad mantiene una exigua representación y no ocupan más que una pequeña parte del área que les pertenece. Fundamentalmente se localiza en los subsectores Marbellí, Malacitano y Axarquiense. La primera etapa de sustitución es un lentiscar (Bupleuro-Pistacietum lentisci). Los matorrales aclarados se incluyen en Teucrio-Coridothymetum y el espartal en Thymo gracile-Stipetum tenacissimae. La alteración del medio ha dado lugar a la proliferación de comunidades nitrófilas, entre otras Inulo-Oryzopsietum miliaceae.

1.2. Faciación acidófila con alcornoques. Este encinar con alcornoques (SmilacoQuercetum rotundifoliae quercetosum suberis) se localiza sobre sustratos paleozoicos y en ecótopos con suficiente compensación edáfica o de ombroclima seco superior de los subsectores Malacitano y Axarquiense. Como primera etapa de sustitución de este encinar-alcornocal, sobre suelos profundos y frescos, se presentan las formaciones arbustivas densas de Bupleuro-Ononidetum speciosae. En zonas de topografía suave, hondonadas, cabeceras de arroyos, entre los 500 y 1200 m. en exposición sur, es posible reconocer unas formaciones densas (escobonales), de posición sintaxonómica incierta, constituidas por Cytisus grandiflorus, Adenocarpus telonensis, Cytisus malacitanus, Retama sphaerocarpa, etc. En los terrenos abandonados y fuertemente 
degradados, en ombroclima seco-subhúmedo, se instala un jaral de LavanduloGenistetum equisetiformis thymetosum capitati. Son frecuentes, así mismo, el espartal de Thymo gracile-Stipetum tenacissimae y los pastizales de Inulo-Oryzopsietum miliaceae.

1.3. Faciación con efedras sobre mármoles dolomíticos. Encinar-coscojar con efedras localizado en el subsector Mijense (Sierra de Mijas) en el piso termomediterráneo (mesomediterráneo). Como primera etapa de sustitución es característica y diferencial la presencia de un coscojar de Rhamno velutini-Quercetum cocciferae. Sobre suelos alterados se desarrolla un jaral de Cisto clusii-Ulicetum baetici, que en suelos de textura franco arenosa se enriquece en Halimium halimifolium. La alteración del medio ha dado lugar a la proliferación de comunidades nitrófilas, entre otras Inulo-Oryzopsietum miliaceae.

1.4. Faciación con bojes sobre mármoles dolomíticos. Encinar con bojes (Smilaco-Quercetum rotundifoliae buxetosum balearicae) localizado en el subsector Almijarense sobre suelos profundos, ombroclima seco superior-subhúmedo en mosaico con las comunidades edafófilas del Cneoro-Buxeto balearicae. Como primera etapa de sustitución se desarrolla un espinar de Bupleuro-Pistacietum lentisci buxetosum balearicae. El matorral de sustitución de este espinar, sobre alfisoles, es el aulagar de Cisto-Ulicetum rivasgodayanii genistetosum retamoidis. Así mismo se encuadran en esta serie los lastonares de Phlomidio-Brachypodietum retusi, espartales de ThymoStipetumm tenacissimae stipetosum giganteae y cerrillares de Aristido-Hyparrhenietum pubescentis. Respecto a las comunidades nitrófilas, es frecuente en los bordes de caminos y carriles el altabacar, Inulo-Oryzopsietum miliaceae.

1.5. Faciación con artos. Este espinar con encinas (Smilaco-Quercetum rotundifoliae maytenetosum europaei) se desarrolla en una estrecha banda litoral comprendida entre Maro y Rincón de la Victoria (subsector Alpujarreño), en ombroclima seco-subhúmedo y con neta influencia litoral. Como primera etapa de sustitución presenta un espinar con Maytenus europaeus (Rhamno-Maytenetum europaei). Como matorral serial aparece, en los alrededores de Maro y Nerja, el tomillar de Odontito-Thymetum baetici que hacia el oeste es sustituido progresivamente por Teucrio-Coridothymetum capitati.

2. Serie mesomediterránea, Bética y Mariánico-Monchiquense, (seca) subhúmeda (húmeda), basófila de la encina (Quercus rotundifolia). Paeonio coriaceae-Querceto rotundifoliae $\mathrm{S}$.

Los encinares climatófilos mesomediterráneos (Paeonio-Quercetum rotundifoliae) están muy bién representados en el territorio. Se reconocen las siguientes faciaciones en los que el ombroclima resulta diferenciador:

2.1. Faciación típica. Este encinar se localiza fundamentalmente en el sector Rondeño. El matorral alto corresponde a Crataego-Quercetum cocciferae, que en zonas 
de precipitaciones altas se enriquece en especies de Lonicero-Berberidion hispanicae. En el subsector Torcalense, sobre calizas y margocalizas, se establece un matorral alto y denso de Ulici parviflori-Genistetum speciosae lavanduletosum lanatae, que es sustituido en el subsector Rondense por Ulici baetici-Lavanduletum lanatae lavanduletosum lanatae. El espartal es de Thymo-Stipetum tenacissimae y los lastonares de suelos degradados son de Phlomidio-Brachypodietum retusi. En medios nitrificados aparece el Artemisio-Santolinetum canescentis.

En el subsector Rondense (Nava de San Luis, Parauta) se localiza una variante sobre suelos con un alto grado de descalcificación caracterizada por la presencia de Quercus suber, Cistus laurifolius, Cytisus grandiflorus, Erica arborea, Erica scoparia, Cistus salvifolius, Lavandula stoechas, etc.

2.2. Faciación con quejigos. Este encinar con quejigos (Paeonio-Quercetum rotundifoliae quercetosum fagineae) se localiza en áreas de ombroclima subhúmedo, húmedo, sobre inceptisoles y terra rossa y tiene su mejor expresión en las laderas norte de la cordillera antequerana (subsector Torcalense). El espinal de sustitución es un coscojar de Crataego-Quercetum cocciferae que en suelos decapitados está reemplazado por el matorral de Ulici-Genistetum speciosae. Este se presenta en mosaico, en los niveles inferiores de este piso, con el espartal de Thymo-Stipetum tenacissimae y, en los niveles superiores, con el lastonar de Helictotricho-Festucetum scariosae. Los pastizales de suelos más degradados se incluyen en el PhlomidioBrachypodietum retusi. En medios nitrificados aparece el Artemisio-Santolinetum canescentis.

2.3. Faciación con lentiscos y escobones. Este encinar (Paeonio-Quercetum rotundifoliae pistacietosum) representa la vegetación potencial de casi toda la parte norte de la provincia (subsector Antequerano). Bioclimáticamente incluible en el piso mesomediterráneo cálido de ombroclima seco, sobre sustratos en su mayoría margosos, en los que no faltan afloramientos de yesos. Como etapas de sustitución aparecen matorrales de Crataego-Quercetum cocciferae y Genisto-Cytisetum fontanesii; en aquellos lugares con litosoles cacalcáreos es frecuente el Teucrio-Coridothymetum capitati. El espartal corresponde a Thymo-Stipetum tenacissimae.

3. Serie supramediterránea, Bética, seca-subhúmeda, basófila de la encina (Quercus rotundifolia). Berberido hispanicae-Querceto rotundifoliae S.

Este encinar con predominio de especies de porte arbustivo y espinosas (Berberido-Quercetum rotundifoliae) se localiza fundamentalmente en el subsector Almijarense. La orla natural es un espinar de Crataego-Loniceretum arboreae. El matorral de salvias que se instala en suelos ligeramente alterados y decapitados corresponde al Convolvulo-Lavanduletum lanatae y los pastizales vivaces son de Helictotricho-Festucetum scariosae, que pueden llegar a formar tapices densos y extensos.

En los subsectores Rondense y Torcalense esta serie adquiere menor relevancia 
debido a la menor continentalidad de estas sierras, que a su vez favorece el desarrollo de los quejigares del Daphno latifoliae-Acereto granatensis quercetosum alpestris y del Paeonio-Querceto rotundifoliae faginetoso con los que comparte sus etapas de sustitución.

4. Serie supra, mesomediterránea, Bética, seca-subhúmeda, silicícola de la encina (Quercus rotundifolia). Adenocarpo-Querceto rotundifoliae S.

Encinar con cenizos y alcornoques (Adenocarpo-Quercetum rotundifoliae quercetosum suberis) puntualmente representado en la provincia de Málaga (subsector Almijarense), entre los 1100 y 1400 m. Como primera etapa de sustitución aparece un piornal de Cytiso-Adenocarpetum decorticantis cytisetosum grandiflorii. Como matorral serial se instala un jaral de Lavandulo-Genistetum equisetiformis cistetosum laurifolii. Los pastizales vivaces estan representados por el lastonar de DactyloFestucetum scariosae.

5. Serie termomediterránea, Gaditano-Onubo-Algarviense, Mariánico-Monchiquense, Bética y norteafricana, subhúmeda y silicícola del alcornoque (Quercus suber). Myrto communis-Querceto suberis $\mathrm{S}$.

Los alcornocales climatófilos termomediterráneos (Myrto communis-Quercetum suberis) están bien representados en la provincia y en función del sustrato podemos reconocer dos faciaciones.

5.1. Faciación típica. Alcornocal localizado fundamentalmente en el subsector Marbellí sobre sustratos paleozoicos (gneises y micaesquistos) alcanzando hasta los 500-600 m. de altitud. Como primera etapa de sustitución se encuentra un madroñal de Cytiso-Arbutetum unedi quercetosum cocciferae, que en zonas de menor desarrollo edáfico o fuerte influencia litoral es reemplazado por un espinar de AsparagoCalicotometum villosae. El jaral de sustitución pertenece al Calicotomo-Genistetum hirsutae. En zonas de acumulación de hojarasca se desarrolla Clinopodio-Origanetum virentis. Es frecuente la comunidad nitrófila de Inulo-Oryzopsietum miliaceae.

5.2. Faciación psammófila. El alcornocal sobre arenales costeros consolidados (Myrto communis-Quercetum suberis halimietosum) se localiza en determinados enclaves del subsector Marbellí. Lleva como etapas de sustitución un monte alto de Asparago-Calicotometum villosae y un jaral que consideramos como un fragmento de asociación del Halimio-Stauracanthetum genistoidis.

6. Serie meso-termomediterránea, Bética, húmeda (hiperhúmeda) silicícola del alcornoque (Quercus suber). Teucrio baetici-Querceto suberis $\mathrm{S}$.

Los alcornocales climatófilos meso y termomediterráneos (Teucrio baetici- 
Querceto suberis) de carácter ombrófilo se localizan en los sectores Aljíbico, Rondeño y Malacitano-Axarquiense. En función de determinadas variaciones climáticas se pueden reconocer tres faciaciones.

6.1. Faciación típica. Alcornocal que se desarrolla sobre las areniscas del Aljibe (subsector Aljíbico) y lleva como primera etapa de sustitución un madroñal de Cytiso-Arbutetum unedi arbutetosum que frecuentemente se acompaña de Clinopodio villosae-Origanetum virentis. Sobre suelos podsolizados se instala la comunidad de quejigos enanos (Phyllireo-Quercetum fruticosae) que en zonas de menor desarrollo edáfico puede comportarse como comunidad permanente. La siguiente etapa de sustitución es un brezal, Genisto tridentis-Stauracanthetum boivinii, que en áreas de mayor alteración es sustituido por una jaral de Ulici borgiae-Cistetum ladaniferi..

6.2. Faciación con quejigo moruno. Alcornocal (Teucrio baetici-Quercetum suberis quercetosum canariensis) desarrollado en áreas de ombroclima húmedo en donde son frecuentes los fenómenos de criptoprecipitación y compensación edáfica. Se localiza en el subsector Aljíbico y sus etapas de sustitución las comparte con la faciación típica.

6.3. Faciación con encinas. Este alcornocal mezclado con encinas (Teucrio baetici-Quercetum suberis quercetosum rotundifoliae) se desarrolla fundamentalmente en el subsector Axarquiense presentando ciertas introgresiones en los sectores Rondeño y Aljíbico. Se caracteriza bioclimáticamente por la existencia de una continentalidad acusada y un descenso de las precipitaciones. Como primera etapa de sustitución se reconoce un madroñal de Cytiso-Arbutetum unedi bupleuretosum fruticosi. que puede ir acompañado de Clinopodio villosae-Origanetum virentis. Los matorrales de sustitución en los subsectores Axarquiense y Rondeño pertenecen al Lavandulo-Genistetum equisetiformis, siendo sustituidos en el sector Aljíbico por brezales del Genisto tridentis-Stauracanthetum boivinii.

7. Serie termo-mesomediterránea, gaditana, húmeda-hiperhúmeda, silicícola del quejigo africano (Quercus canariensis). Rusco hypophylli-Querceto canariensis S.

Quejigal localizado en el sector Aljíbico en áreas meso-termomediterráneas, húmedo-hiperhúmedas, sobre alfisoles ácidos y humificados. Sus etapas seriales son idénticas a las del alcornocal de Teucrio-Querceto suberis, con el que entra siempre en contacto catenal (Cytiso-Arbutetum unedi arbutetosum unedi, GenistoStauracanthetum boivinii).

8. Serie supra, mesomediterránea, Bética, subhúmeda-húmeda, basófila del quejigo (Quercus faginea). Daphno latifoliae-Acereto granatensis S.

Quejigal localizado en el sector Rondeño y en el subsector Almijarense. En función de distintas características climáticas se reconocen dos faciaciones.

8.1. Faciación típica. Quejigal de areal eminentemente Subbético alcanza 
puntual y fragmentariamente, en áreas microclimáticas, el subsector Almijarense. La alteración edáfica da lugar a una moderada erosión superficial que favorece la entrada de especies y comunidades pertenecientes a la serie del Berberidi-Querceto rotundifoliae, con la que comparte las etapas de sustitución.

8.2. Faciación con Quercus alpestris. Se localiza en el Sector Rondense (de menor continentalidad que Sierra Tejeda). Este quejigar (Daphno latifoliae-Aceretum granatensis quercetosum alpestris*) va acompañado de las siguientes etapas de sustitución: un espinal de Pruno mahalebo-Berberidetum hispanicae y un matorral xeroacántico de Ulici baetici-Lavanduletum lanatae erinaceetosum. Otras comunidades seriales corresponden a los lastonares de Helictotricho-Festucetum scariosae y los matorrales subnitrófilos de Artemisio-Santolinetum canescentis.

9. Serie supra, mesomediterránea, Rondense, basófila del pinsapo (Abies pinsapo). Paeonio broteroi-Abieteto pinsapo $\mathrm{S}$.

De areal eminentemente Rondeño (subsector Rondense), el pinsapar (Paeonio broteroi-Abietetum pinsapo) se localiza en áreas de ombroclima húmedo. Como primera etapa de sustitución se reconoce un espinal de Pruno mahalebo-Berberidetum hispanicae. El matorral serial, en el piso supramediterráneo es de Lavandulo-Ulicetum baetici erinaceetosum. Los lastonares son de Helictotricho-Festucetum scariosae y los matorrales subnitrófilos de Artemisio-Santolinetum canescentis. En las partes basales, mesomediterráneas, el abetal se pone en contacto con el encinar, participando de sus etapas de sustitución.

10. Serie supramediterránea, Bética, subhúmeda, silicícola del roble melojo (Quercus pyrenaica). Adenocarpo-Querceto pyrenaicae $\mathrm{S}$.

Robledal localizado exclusivamente en el subsector Almijarense en altitudes comprendidas entre los 1500 y $1600 \mathrm{~m}$. La primera etapa de sustitución, en suelos poco alterados, está constituida por el piornal de Cytiso-Adenocarpetum decorticantis, y en suelos más degradados por el jaral de Lavandulo-Genistetum equisetiformis cistetosum laurifolii. Aparecen también comunidades nitrófilas camefíticas de Artemisio barrelieri-Helichrysetum serotini y lastonares de Dactylo-Festucetum scariosae.

Daphno latifoliae-Aceretum granatensis Rivas-Martínez 1964 quercetosum alpestris subass. nov.*. Como inventario tipo damos a conocer el efectuado en la Sierra de las Nieves, Tolox (Málaga), 1700 m., cobertura del $80 \%$, exposición norte y área de $200 \mathrm{~m} 2$. Características de la subasociación: Quercus alpestris, 3; Sorbus aria, +; Daphne laureola, 1; Acer granatensis, +. Características de unidades superiores: Berberis hispanica, 3; Prunus prostrata, $1 ;$ Rhamnus saxatilis, 2; Helleborus phoetidus, 1; Crataegus monogyna, 1. Compañeras: Astragalus sempervirens, 1; Abies pinsapo, +; Juniperus sabina, +; Bupleurum spinosum, +; Erinacea anthyllis, +; Erodium cheilanthifolium, +; Cerastium gibraltaricum, + . 
Manifestaciones puntuales de Quercus pyrenaica aparecen en los subsectores Algíbico y Rondense, en contacto con alcornocales.

11. Serie oromediterránea, Bética, subhúmeda-húmeda, basófila de la sabina rastrera (Juniperus sabina). Daphno oleoidi-Pineto sylvestris $\mathrm{S}$.

Ocupa las partes más elevadas, por encima de 1800 m., de las cordilleras malagueñas, subsectores Rondense y Almijarense. La etapa madura es un enebral de porte rastrero, Daphno-Pinetum sylvestris, en el que es de resaltar la ausencia de Daphne oleoides. Sobre las grietas anchas de los lapiaces y litosuelos se instala el matorral de Festuco-Astragaletum granatensis. Son frecuentes los pastizales de Minuartio-Poion ligulatae.

\section{Series y complejos de comunidades edafófilas.}

Son numerosas las referencias a comunidades de tal naturaleza en la provincia de Málaga: Cabezudo, Nieto Caldera y Pérez Latorre (1989), López González (1975), Martínez Parras y Esteve (1980); Martínez Parras, Peinado y Alcaraz (1986); Molero y Pérez Raya (1987); Nieto Caldera (1987), Nieto Caldera, Pérez Sanz y Cabezudo (1987); Nieto Caldera, Cabezudo y Trigo (1989), Pérez Raya (1987), Rivas Goday (1969; 1973); Rivas Goday y Esteve (1972); Rivas Goday y López González (1979); Rivas-Martínez, Izco y Costa (1973), etc. En general son unidades de vegetación constituidas por complejos de comunidades que presentan una relación dinámica incierta. Las series y complejos de comunidades presentes en la provincia son las siguientes:

G. Sabinares y pinares.

12. Geoserie psammófila.

13. Chamaeropo-Junipereto phoeniceae $S$.

14. Rhamno myrtifolii-Junipereto phoeniceae S.

15. Cneoro tricoci-Buxeto balearici $\mathrm{S}$.

16. Pino pinastri-Querceto cocciferae $S$.

H. Pinsapar serpentinícola.

17. Bunio macucae-Abieteto pinsapi $S$.

I. Acebuchal.

18. Tamo comunis-Oleeto sylvestris $S$.

12. Geoseries psammófilas. En los arenales próximos a Marbella (subsector Marbellí) es posible reconocer el complejo de comunidades siguiente. Sobre sustratos ya consolidados y fijados se instala el sabinar de Osyrio-Juniperetum turbinatae que como matorral de sustitución lleva un jaral de Halimio-Stauracanthetum genistoidis. El pastizal es de Malcolmio-Vulpietum alopecuri. Las dunas móviles están colonizadas 
por diferentes comunidades: Otantho-Ammophiletum, Eryngio-Elymetum, OnonidiLinarietum pedunculatae y Sporobolo arenarii-Agropyretum farcti.

13. Serie edafoxerófila, calcícola, termomediterránea (meso inferior), seca, subhúmeda de la sabina (Juniperus phoenicea). Chamaeropo-Junipereto phoeniceae $\mathrm{S}$.

Los sabinares edafófilos termomediterráneos calcícolas alcanzan una gran diversidad en la provincia de Málaga, pudiendo diferenciar dos faciaciones.

13.1. La faciación típica de dicho sabinar se localiza en los subsectores Rondense y Torcalense sobre sustratos calizos y protosuelos. Lleva como matorral serial Teucrio-Coridothymetum capitati. Es frecuente la comunidad nitrófila de Inulo-Oryzopsietum miliaceae.

13.2. Faciación con piornos. Localizada fundamentalmente en el subsector Mijense (Sierra Blanca, Marbella). Se define por la presencia de Genista haenseleri (Chamaeropo-Juniperetum phoeniceae genistetosum haenseleri*) que sobre litosuelos se acompaña de un matorral aclarado de Coridothymo-Genistetum haenseleri y en suelos más profundos por Cisto-Ulicetum baetici. En lugares de influencia humana es frecuente la comunidad nitrófila de Inulo-Oryzopsietum miliaceae.

14. Serie edafoxerófila calcícola, Bética, meso y supramediterránea, subhúmeda de la sabina (Juniperus phoenicea). Rhamno myrtifolii-Junipereto phoeniceae S.

Sabinares edafófilos localizados exclusivamente sobre arenales y roquedos calizo-dolomíticos de los subsectores Rondense y Almijarense.

El sabinar (Rhamno myrtifolii-Juniperetum phoeniceae) es una comunidad de carácter permanente y de aspecto fisiognómico variable, ya que pueden resultar dominantes Pinus pinaster o Pinus halepensis. Los matorrales y pastizales que se desarrollan en estos biotopos varían entre los incluibles en las asociaciones Centaureo bombycinae-Lavanduletum lanatae, Helianthemo-Anthyllidetum argyrophyllae y Brachypodio boissieri-Trisetetum velutini (subsector Almijarense) y los UliciLavanduletum lanatae y Thymo gracile-Stipetum tenacissimae de los subsectores Rondense y Torcalense.

Chamaeropo humilis-Juniperetum phoeniceae Rivas-Martínez 1989 genistetosum haenselerii subass. nov*. Como inventario tipo damos a conocer el efectuado en la Sierra Blanca, Istán (Málaga), 600 m., cobertura del $70 \%$, exposición norte y área de $200 \mathrm{~m} 2$. Características de la subasociación: Juniperus phoenicea, 3; Genista haenseleri, 3; Ephedra fragilis, 1. Características de unidades superiores: Juniperus oxycedrus, 2; Osyris cuadripartita, 1; Chamaerops humilis, $2 ;$ Asparagus albus, $1 ;$ Daphne gnidium, $1 ;$ Ceratonia siliqua, $+;$; Pistacia lentiscus, $1 ;$ Pistacia terebinthus, $+;$ Rhamnus oleoides,$+;$ Rubia peregrina,$+;$ Olea europaea, $+;$ Bupleurum gibraltaricum, + ; Smilax aspera, + ; Lonicera implexa, + ; Asparagus stipularis, +; Jasminum fruticans, +. Compañeras: Phlomis purpurea, 1; Cistus albidus, 1; Thymbra capitata , +; Stipa tenacissima, 1; Mercurialis tomentosa; Thymus baeticus, 1; Ulex baeticus, 1; Satureja obovata, 1; Helichrysum serotinum, +; Rosmarinus officinalis, +; Brachypodium ramosum, 3; Arisarum vulgare, 1; Phlomis lychnitis, 1; Paronychia suffruticosa, 1. 
15. Serie edafoxerófila-dolomitícola, Almijarense, termo y mesomediterránea inferior, seca superior y subhúmeda del boj (Buxus balearica). Cneoro-Buxeto balearici $\mathrm{S}$.

Los mármoles dolomíticos kakiritizados de la Sierra Almijara (subsector Almijarense) que se extienden desde el nivel del mar hasta los $1000 \mathrm{~m}$. de altitud muestran un tapiz vegetal claramente individualizado florísticamente por la presencia de algunos elementos relícticos, como Buxus balearica y Cneorun triccocum..

La asociación considerada como cabecera de serie es el Cneoro-Buxetum balearici en la que Pinus halepensis puede originar un estrato arbóreo de elevada cobertura. En condiciones favorables (disminución de pendientes y mayor desarrollo edáfico) entra en contacto con el Smilaci-Quercetum rotundifoliae buxetosum balearici. En la degradación de esta bojeda se instalan un aulagar, Cisto clusii-Ulicetum (parviflori) rivasgodayanii, un espartal de Thymo gracile-Stipetum tenacissimae stipetosum giganteae y un lastonar de Brachypodio-Trisetetum velutini. Son frecuentes las comunidades nitrófilas de Inulo-Oryzopsietum miliaceae y AristidoHyparrhenietum.

16. Serie serpentinícola, Bermejense, termo y mesomediterránea, seca, subhúmeda, húmeda del pino negral (Pinus pinaster). Pino pinastri-Querceto cocciferae S.

Coscojar con pinos (Pino pinastri-Quercetum cocciferae) desarrollado sobre suelos en roca madre serpentínica (sector Bermejense). Los matorrales de sustitución se incluyen en la asociación Halimio-Digitaletum laciniatae. Otras comunidades presentes en estos medios son Arenario-Iberidetumfontqueri y Asplenio-Saxifragetum gemmulosae. En lugares basales es frecuente la comunidad nitrófila de InuloOryzopsietum miliaceae.

17. Serie serpentinícola, Bermejense, supra-mesomediterránea, (subhúmeda) húmeda del pinsapo (Abies pinsapo). Bunio macucae-Abieteto pinsapi $\mathrm{S}$.

Pinsapar, Bunio macucae-Abietetum pinsapi, localizado sobre los afloramientos serpentínicos de la sierra Bermeja (subsector Bermejense). Las etapas seriales son comunes con las del Pino-Querceto cocciferae, donde posiblemente podría incluirse como faciación del mismo.

18. Serie termomediterránea bético-gaditana subhúmeda-húmeda, verticícola del acebuche (Olea sylvestris). Tamo comunis-Oleeto sylvestris $\mathrm{S}$.

En la zona suroccidental de la provincia de Málaga (subsector Aljíbico) y sobre vertisoles se presenta como vegetación potencial una formación arbórea dominada por el acebuche (Tamo-Oleetum sylvestris). En sus etapas seriales se pueden identificar 
un espinar de Asparago-Rhamnetum oleoidis y un matorral de Asperulo hirsutiUlicetum scabri.

En el subsector Antequerano es característica la presencia, en relieves calizos de fuertes pendientes, litosuelos y con una elevada insolación (exposición sur), de unas formaciones dominadas por el acebuche e incluibles provisionalmente en Asparago-Rhamnetum oleoidis.

\section{ESQUEMA SINTAXONÓMICO DE LAS COMUNIDADES MENCIONADAS EN EL TEXTO}

AMMOPHILETEA ARENARIAE BR.-BL. \& R.TX. 1933

Ammophiletalia (arundinaceae) BR.-BL. (1931) 1933

Ammophilion arundinaceae Géhu 1986/88

Eryngio maritimi-Elymetum farcti Géhu (1986) 1988

Otantho-Ammophyletum arundinaceae Géhu, Rivas-Martínez \& R. Tx. In Géhu 1975

Agropyrion junceiformis (R. Tx. in Br.-Bl. \& R. Tx. 1952) Géhu, Rivas-Martínez \& R. Tx. 1972

Sporobolo arenarii-Agropyretum farcti (Br.-Bl. 1933) Rivas-Martínez y Costa 1980

PEGANO-SALSOLETEA Br.-Bl. \& O. Bolós 1957

Helicryso-Santolinetalia Peinado y Martínez Parras 1984

Santolinion pectinato-canescentis Peinado y Martínez Parras 1984

Artemisio glutinosae-Santolinetum canescentis Peinado y Martínez Parras 1984

Artemisio-Santolinion Costa 1975

Artemisio barrelieri-Helichrysetum serotini (Rivas Goday y Esteve 1972)

Peinado, Martínez Parras y C. Bartolomé 1986

TUBERARIETEA GUTTATAE Br.-B1. 1952 em. Rivas-Martínez 1978

Trachynietalia distachyae Rivas-Martínez 1978

Omphalodion linifoliae Rivas-Martínez, Izco y Costa 1973

Omphalodenion brassicifoliae Pérez Raya 1987 Arenario capillipes-Iberidetum fontqueri Rivas-Martínez, Izco y Costa 1973

Silenenion germanicae Pérez Raya 1987

Jasiono penicillatae-Linarietum saturejoidis Rivas-Martínez, Izco y Costa 1973

Tuberarietalia guttatae Br.-Bl. 1940 em. Rivas-Martínez 1978

Tuberarion guttatae Br.-B1. 1940

Malcolmietalia Rivas Goday 1957

Anthyllido-Malcolmion lacerae Rivas Goday 1957 em. Rivas-Martínez 1978 Malcolmio-Vulpietum alopecuri Díez Garretas, Hernández y Asensi 1975

Linarion pedunculatae Díez Garretas, Asensi y Esteve 1977

Ononidi variegatae-Linarietum pedunculatae Díez Garretas, Asensi y Esteve 1977

LYGEO SPARTI-STIPETEA TENACISSIMAE Rivas-Martínez 1977

Lygeo-Stipetalia (Br.-Bl. \& O. Bolós (1954) 1957) em. Rivas-Martínez 1977

Stipion tenacissimae Rivas-Martínez 1977

Thymo gracile-Stipetum tenacissimae Pérez Raya 1987 stipetosum giganteae Nieto Caldera y Cabezudo 1988

Festucion scariosae Martínez Parras, Peinado y Alcaraz 1983

Helictotricho filifolii-Festucetum scariosae Martínez Parras, Peinado y Alcaraz 1983 
Dactylo hispanicae-Festucetum scariosae Martínez Parras, Peinado y Alcaraz 1987

Phlomidio lychnitidis-Brachypodion retusi G.Mateo 1984

Phlomidio lychnitidis-Brachypodietum retusi Br.-Bl. \& O. Bolós 1957

Brachypodio boissieri-Trisetetum velutini Martínez Parras, Peinado y Alcaraz 1986

Hyparrhenietalia hirtae Rivas-Martínez 1978

Saturejo-Hyparrhenion hirtae O. Bolós 1962

Aristido coerulescens-Hyparrhenietum pubescentis Rivas-Martínez ex Alcaraz 1984

Bromo-Oryzopsion miliaceae (A. y O. Bolós 1950) O. Bolós 1957

Inulo viscosae-Oryzopsietum miliaceae (A. y O. Bolós 1950) O. Bolós 1957

CALLUNO-ULICETEA Br.-Bl. \& R. Tx. 1943

Calluno-Ulicetalia (Quantin 1935) R. Tx. 1937 em. Rivas-Martínez 1979

Ericion umbellatae Br.-Bl., Pinto, Rozeira \& Fortes 1952

Stauracanthenion boivinii Rivas-Martínez 1979

Genisto tridentis-Stauracanthetum boivinii Rivas-Martínez 1979

FESTUCO HYSTRICIS-ONONIDETEA STRIATAE Rivas-Martínez, Díaz, Fernández Prieto, Loidi y Penas 1991

Festuco-Poetalia ligulatae Rivas Goday y Rivas-Martínez 1963

Minuartio-Poion ligulatae O. Bolós 1962

Seselido granatensis-Festucetum hystricis Martínez Parras, Peinado y Alcaraz 1984

Erodio daucoides-Saxifragetum erioblastae Pérez Raya y Losa Quintana 1986

ROSMARINETEA OFFICINALIS Br.-Bl. 1947 em. Rivas-Martínez, Díaz, Fernández

Prieto, Loidi y Penas 1991

Erinacetalia anthyllidis Quézel 1951

Xeroacantho-Erinaceion Quézel 1951 O. Bolós 1967

Festuco hystricis-Astragaletum boissieri Quézel 1953

Pterocephaletalia spathulati Rivas-Martínez, Pérez Raya y Molero 1987

Andryalion agardhii Rivas-Martínez 1961

Helianthemo visciduli-Anthyllidetum argyrophyllae Rivas Goday y Esteve 1972

Rosmarinetalia Br.-Bl. (1931) 1952

Lavandulo lanatae-Echinospartion boissieri Rivas Goday y Rivas-Martínez 1968

Centaureo bombycinae-Lavanduletum lanatae (Rivas Goday y Esteve 1972)

Martínez-Parras, Peinado y Alcaraz 1983

Ulici baetici-Lavanduletum lanatae Martínez Parras, Peinado y De La Cruz 1987 lavanduletosum lanatae erinaceetosum anthyllidis Martínez Parras, Peinado y De La Cruz 1987

Convolvulo lanuginosi-Lavanduletum lanatae Rivas Goday y Rivas-Martínez 1968

Saturejo-Coridothymion Rivas Goday y Rivas-Martínez 1968

Cisto clusii-Ulicetum baetici Nieto Caldera, Pérez Sanz y Cabezudo 1987 ulicetosum baetici

thymetosum capitati Nieto Caldera, Pérez Sanz y Cabezudo 1987

teucrietosum chrysotrici Nieto Caldera, Pérez Sanz y Cabezudo 1987

Teucrio lusitanici-Coridothymetum capitati Rivas Goday y Rivas-Martínez 1968

Cisto clusii-Ulicetum (parviflorii) rivasgodayanii Nieto Caldera y Cabezudo 1988 ulicetosum rivasgodayanii

genistetosum retamoidis Nieto Caldera y Cabezudo 1988

anthyllidetosum plumosae Nieto Caldera y Cabezudo 1988

odontitosum purpureae Nieto Caldera y Cabezudo 1988

Odontito purpureae-Thymetum baetici Esteve y López Guadalupe 1977 teucrietosum eriocephali Martínez Parras y Esteve 1980

Genisto umbellatae-Cytisetum fontanesii Rivas Goday y Rivas-Martínez 1968 
Ulici parviflori-Genistetum speciosae Rivas Goday y Rivas-Martínez 1968 lavanduletosum lanatae Rivas Goday y Rivas-Martínez 1968

Odontito purpureae-Thymetum baetici López Guadalupe y Esteve 1977

Coridothymo capitati-Genistetum haenseleri Rivas Goday y Rivas-Martínez 1968

CISTO-LAVANDULETEA Br.-Bl. (1940) 1952

Lavanduletalia stoechidis Br.-Bl. 1940 em. Rivas-Martínez 1968

Stauracantho-Halimion halimifolii Rivas-Martínez 1979

Halimio-Stauracanthetum genistoidis Rivas-Martínez, Costa, Castroviejo y Valdés 1980

Ulici argentei-Cistion ladaniferi (Br.-Bl. 1940) Br.-Bl., Silva \& Rozeira 1964 em. RivasMartínez 1979

Lavandulo-Genistetum equisetiformis Rivas Goday y Rivas-Martínez 1968 genistetosum umbellatae

thymetosum capitati Martínez Parras, Peinado y Alcaraz 1985

cistetosum laurifolii Martinez Parras, Peinado y Alcaraz 1987

Calicotomo villosae-Genistetum hirsutae Martínez Parras, Peinado y De la Cruz

1987

Ulici borgiae-Cistetum ladaniferi Asensi y Díez Garretas 1987

Asperulo hirsutae-Ulicetum scabri Rivas-Martínez 1987

Staehelino-Ulicion baetici Rivas Goday y Rivas-Martínez 1967

Halimio atriplicifolii-Digitaletum laciniatae Rivas Goday y Rivas-Martínez 1967

CYTISETEA SCOPARIO-STRIATI Rivas-Martínez 1974

Cytisetalia scopario-striati Rivas-Martínez 1974

Genistion floridae Rivas-Martínez 1974

Adenocarpenion decorticantis Valle 1981

Cytiso scopari-Adenocarpetun decorticantis Valle 1981 adenocarpetosum decorticantis

cytisetosum grandiflorii Mota y Valle 1987

PINO-JUNIPERETEA Rivas-Martínez 1964

Pino-Juniperetalia Rivas-Martínez 1964

Juniperenalia sabinae Rivas-Martínez 1986

Pino-Juniperion sabinae Rivas Goday (1956) 1960

Daphno oleoidi-Pinetum sylvestris Rivas-Martínez 1964

QUERCO-FAGETEA Br.-BI. \& Vlieger in Vlieger 1937

QUERCO PETRAEAE-FAGENEA SYLVATICAE Rivas-Martinez y Cantó 1987

Quercetalia pubescenti-petraeae Klika corr. Moravec in Begyuin \& Theurillat 1984 nom. mut.

Aceri granatensi-Quercion fagineae (Rivas Goday, Rigual y Rivas-Martínez 1959) RivasMartínez 1987

Daphno latifoliae-Aceretum granatensis Rivas-Martínez 1964

aceretosum granatensis quercetosum alpestris subass. nov.

Paeonio broteroi-Abietetum pinsapo Asensi y Rivas-Martínez 1976

Bunio macucae-Abietetum pinsapo (Asensi y Rivas-Martínez 1976) Rivas-

Martínez 1987

Quercetalia roboris R. Tx. 1931

Quercion robori-pyrenaicae (Br.-Bl., Silva y Rozeira 1959) Rivas-Martínez 1975.

Quercenion pyrenaicae Rivas-Martínez 1975

Adenocarpo decorticantis-Quercetum pyrenaicae Martínez Parras y Molero 1982

RHAMNO-PRUNENEA SPINOSAE (Rivas Goday y Borja 1961) Rivas-Martínez, Arnaiz

y Loidi in Arnaiz y Loidi 1983

Prunetalia spinosae R.Tx. 1952

Lonicero arboreae-Berberidion hispanicae O. Bolós 1954 
Lonicero arboreae-Berberidenion hispanicae Arnaiz 1979

Pruno mahalebo-Berberidetum hispanicae Asensi y Rivas-Martínez 1979

Crataego monogynae-Loniceretum arboreae O. Bolós 1954

TRIFOLIO-GERANIENEA SANGUINEI (T.H. Muller 1962) Rivas-Martínez y Cantó

em. Rivas-Martínez 1987

Origanetalia vulgaris Th. Muller 1961

Origanion virentis Rivas-Martínez y O. Bolós in Rivas-Martínez et al. 1984

Clinopodio villosae-Origanetum virentis Rivas-Martínez in Rivas-Martínez et al. 1984

QUERCETEA ILICIS Br.-Bl. 1947

Quercetalia ilicis Br.-Bl. (1931) 1936 em. Rivas-Martínez 1975

Querco rotundifoliae-Oleion sylvestris Barbero, Quézel y Rivas-Martínez in Rivas-

Martínez, Costa e Izco 1986

Tamo comunis-Oleetum sylvestris Benabid 1984

Rusco-Quercetum canariensis Rivas-Martínez 1975

Smilaco mauritanicae-Quercetum rotundifoliae Barbero, Quézel \& Rivas-Martínez 1981

quercetosum suberis Nieto Caldera, Pérez Latorre y Cabezudo 1990

buxetosum balearicae Martínez Parras, Peinado y Alcaraz 1985

maytenetosum europaei Rivas-Martínez 1985

Teucrio baetici-Quercetum suberis Rivas-Martínez ex Díez Garretas, Cuenca y

Asensi 1988

quercetosum suberis

quercetosum canariensis Díez Garretas, Cuenca y Asensi 1988

quercetosum rotundifoliae Nieto Caldera, Cabezudo y Pérez Latorre 1990

Myrto communis-Quercetum suberis Barbero, Quézel \& Rivas-Martínez 1981 quercetosum suberis

halimietosum Pérez Latorre inéd.

Quercion broteroi Br.-Bl., P. Silva \& Rozeira 1956 em. Rivas-Martínez 1975 corr. V.

Fuente 1986

Paeonio broteroi-Quercenion rotundifoliae Rivas-Martínez in Rivas-Martínez, Costa e

Izco 1986

Paeonio-Quercetum rotundifoliae Rivas-Martínez 1964

quercetosum rotundifoliae

quercetosum fagineae Rivas-Martínez 1964 em. Rivas Goday y Rivas-

Martínez 1971

pistacietosum lentisci Martínez Parras, Peinado y Alcaraz 1986

Berberido hispanicae-Quercetum rotundifoliae Rivas-Martínez 1987

Adenocarpo decorticantis-Quercetum rotundifolae Rivas-Martínez 1987

quercetosum suberis Rivas-Goday y Rivas-Martínez ex Martínez Parras,

Peinado y Alcaraz 1987

Pistacio lentisci-Rhamnetalia alaterni Rivas-Martínez 1975

Asparago albi-Rhamnion oleoidis Rivas Goday 1964 ex. Rivas-Martínez 1975

Asparago aphylli-Calicotometum villosae Rivas-Martínez 1975

Bupleuro gibraltarici-Pistacietum lentisci Martínez Parras, Peinado y Alcaraz

1986

buxetosum balearicae Nieto Caldera y Cabezudo 1988

Chamaeropo humilis-Juniperetum phoeniceae Rivas-Martínez 1989

genistetosum haenselerii subass. nov.

Asparago albi-Rhamnetum oleoidis Rivas Goday 1959

Bupleuro gibraltarici-Ononidetum speciosae Rivas Goday y Rivas-Martínez 1968

Cneoro tricocci-Buxetum balearici Rivas Goday y Rivas-Martínez 1969

Pino pinastri-Quercetum cocciferae Cabezudo, Nieto Caldera y Pérez Latorre

1989

Rhamno velutini-Quercetum cocciferae Nieto Caldera, Pérez Sanz y Cabezudo 1987 
Ericion arboreae (Rivas-Martínez ex Rivas-Martínez, Costa e Izco 1984) Rivas-Martínez 1987

Cytiso baetici-Arbutetum unedonis Nieto Caldera, Pérez Latorre y Cabezudo 1990 arbutetosum quercetosum cocciferae Nieto Caldera, Pérez Latorre y Cabezudo 1990 bupleuretosum fruticosae Pérez Latorre ined.

Juniperion turbinatae Rivas-Martínez 1975 corr. 1987

Osyrio quadripartitae-Juniperetum turbinatae Rivas-Martínez 1988

Rhamno oleoidi-Quercion fruticosae Barbero, Quézel \& Rivas-Martínez ex Rivas-

Martínez, Costa e Izco 1984

Phyllireo angustifoliae-Quercetum fruticosae Barbero, Quézel \& Rivas-Martínez 1981

Rhamno lycioidis-Quercion cocciferae (Rivas Goday 1964) Rivas-Martínez 1975

Rhamno myrtifolii-Juniperetum phoeniceae Molero y Pérez Raya 1987

Crataego monogynae-Quercetum cocciferae Martínez Parras, Peinado y Alcaraz 1984

\section{BIBLIOGRAFIA}

ASENSI, A. y S. RIVAS-MARTINEZ -1976-Contribución al conocimiento de los pinsapares de la Serranía de Ronda. Inst. Bot. Cavanilles, 33: 239-247.

ASENSI, A. y B. DIEZ GARRETAS -1988- Matorrales y jarales del sector Rondeño. Documents Phytosociologiques, 11: 263-274

CABEZUDO, B., J.M. NIETO CALDERA y A. PÉREZ LATORRE -1989- Contribución al conocimiento de la vegetación edafófilo-serpentinícola del sector Rondeño (Málaga, España). Acta Bot. Malacitana, 14: 291-294.

CEBALLOS, L. y C. VICIOSO -1933- Estudio sobre la vegetación y flora forestal de la provincia de Málaga. Instituto Forestal de Invest. y Exp. Madrid. 285 pgs.

DE LA ROSA, D. y J.M. MOREIRA -1987-Evaluación ecológica de recursos naturales de Andalucía. Servicio de Evaluación de Recursos naturales, Agencia de Medio Ambiente. Memoria y 4 Mapas $1 / 400.000$. Sevilla.

DIEZ, B.; J. CUENCA y A. ASENSI -1988- Datos sobre la vegetación del subsector algíbico (prov. gaditano-onubo-algarviense). Lazaroa, 9: 315-333.

LOPEZ GONZALEZ, G. -1975- Contribución al estudio florístico y fitosociológico de la $\mathrm{S}^{\mathrm{a}}$ de Aguas. Acta Bot. Malacitana, 1: 81-205.

MARTINEZ PARRAS, J.M. y F. ESTEVE -1980- Nuevas comunidades vegetales del sur de la provincia de Granada. Anales Inst. Bot. Cavanilles, 35: 199-218.

MARTINEZ PARRAS, J.M.; J.M. PEINADO y F. ALCARAZ -1986- Sobre la vegetación termófila de la cuenca mediterránea de granada y sus áreas limítrofes. Lazaroa, 8: 251 268.

MARTÍNEZ PARRAS, J.M.; M. PEINADO LORCA y M. DE LA CRUZ ROT -1987Aportación al estudio fitosociológico de los matorrales del sector Rondeño. Studia Botanica, 6: $39-45$.

MOLERO, J. y F. PEREZ RAYA -1987- Estudio fitosociológico de los sabinares de Juniperus phoenicea L. en el sector Malacitano-Almijarense (provincia corclógica Bética). Lazaroa, 7: 301-306.

NIETO CALDERA, J.M., S. PEREZ SANZ y B. CABEZUDO -1987- Datos sobre la vegetación dolomitícola del Sector Rondeño. Lazaroa, 10: 35-46.

NIETO CALDERA, J.M. -1987- Estudio fitocenológico de las sierras Tejeda y Almijara. 
Tesis Doctoral. Univ. Málaga.№ 8, Microfichas.

NIETO CALDERA, J.M. y B. CABEZUDO -1988- Series de vegetación climatófilas de las sierras Tejeda y Almijara (Málaga-Granada). Acta Bot. Malacitana, 13: 229-260.

NIETO CALDERA, J.M.; B. CABEZUDO Y M. TRIGO -1989- SERIES DE VEGETACIÓN EDAFÓFILAS DE LAS SIERRAS TEJEDA Y ALMIJARA (Málaga-Granada, España). Acta Bot. Malacitana, 14: 161-170.

NIETO CALDERA, J.M.; A. PÉREZ LATORRE y B. CABEZUDO -1990- Datos sobre la vegetación silicícola de Andalucía, I. Acta Bot. Malacitana, 14: 179-192.

PEINADO LORCA, M. y S. RIVAS-MARTÍNEZ, eds. -1987- La vegetación de España. Madrid.

PEREZ RAYA, F. -1987- La vegetación en el sector Malacitano Almijarense de Sa Nevada. Tesis Doctoral. Univ. Granada.

RIVAS GODAY, S. -1969- Flora serpentinicola española, nota 1ª . Anales Real Acad. Farmacia, 35(3).

RIVAS GODAY, S. y F. ESTEVE - 1972- Flora serpentinícola española. Anales Real Acad. Farmacia, 38(3): 409-462.

RIVAS GODAY, S. -1973-Plantas serpentinícolas y dolomitícolas del sur de España. Bol Soc Brot. (2 $2^{\mathrm{a}}$ ser.), 47(2): 161-178.

RIVAS GODAY, S. y G. LOPEZ GONZALEZ -1979- Nuevos edafismos hispanos de sustratos ultrabásicos y dolomíticos. Anales Real Acad. Farmacia, 45: 95-112.

RIVAS-MARTINEZ, S. -1985- Biogeografía y vegetación. Madrid.

RIVAS-MARTINEZ, S. -1987- Memoria del mapa de las series de vegetación de España. Madrid.

RIVAS-MARTINEZ, S. -1988- Bioclimatología, biogeografía y series de vegetación de Andalucía Occidental. Lagascalia 15(Extra): 91-119.

RIVAS-MARTINEZ, S.; J. IZCO y M. COSTA -1973- Asplenium cuneifolium Viv. en Sierra Bermeja (Málaga). Comentarios sobre la vegetación y flora serpentinícola y dolomitícola. Trab. Dep. Bot. y F. Veg., 6: 23-30.

RIVAS-MARTÍNEZ, S.; J.C. BASCONES; T.E. DÍAZ; F. FERNÁNDEZ GONZÁLEZ y J. LOIDI -1991- Vegetación del Pirineo Occidental y Navarra. Itinera Geobotánica, 5: 5456.

SAINZ OLLERO, H. y J.E. HERNÁNDEZ BERMEJO - 1985-'Sectorización fitogeográfica de la Península Ibérica e Islas Baleares: la contribución de su endemoflora como criterio de semejanza. Candollea, 40: 485-508.

SALVO, A.E.; J.M. NIETO CALDERA; F. CONDE; J. GUERRA y B. CABEZUDO -1983Especies vegetales endémicas y amenazadas de la provincia de Málaga. Jábega, 44: 6676.

SALVO, A.E. y B. CABEZUDO -1984- Bases para la utilización de los pteridófitos en el establecimiento de unidades corológicas. I, Andalucía. Anales de Biología, 1: 309-316.

(Aceptado para su publicación en junio de 1991)

Dirección de los autores: Departamento de Biología Vegetal. Facultad de Ciencias. Universidad de Málaga. 29080 Málaga. 